Analytical Methods

\title{
Effects of malaxation temperature and harvest time on the chemical characteristics of olive oils
}

\author{
Olusola Samuel Jolayemi, Figen Tokatli*, Banu Ozen \\ Izmir Institute of Technology, Department of Food Engineering, 35430 Urla-Izmir, Turkey
}

\section{A R T I C L E I N F O}

\section{Article history:}

Received 12 February 2016

Received in revised form 14 May 2016

Accepted 20 May 2016

Available online 20 May 2016

\section{Keywords:}

Olive oil composition

Malaxation temperature

Harvest time

Phenolic profile

Fatty acid profile

Oxidative stability

Classification

Multivariate regression

\begin{abstract}
A B S T R A C T
The aim of the study was to determine the effects of harvest time and malaxation temperature on chemical composition of olive oils produced from economically important olive varieties with a full factorial experimental design. The oils of Ayvalik and Memecik olives were extracted in an industrial two-phase continuous system. The quality parameters, phenolic and fatty acid profiles were determined. Harvest time, olive variety and their interaction were the most significant factors. Malaxation temperature was significant for hydroxytyrosol, tyrosol, p-coumaric acid, pinoresinol and peroxide value. Early and midharvest oils had high hydroxytyrosol and tyrosol (maximum $20.7 \mathrm{mg} / \mathrm{kg}$ ) and pigment concentrations (maximum chlorophyll and carotenoids as $4.6 \mathrm{mg} / \mathrm{kg}$ and $2.86 \mathrm{mg} / \mathrm{kg}$, respectively). Late harvest oils were characterized with high peroxide values $\left(9.2-25 \mathrm{meq} \mathrm{O}_{2} / \mathrm{kg}\right)$, stearic (2.4-3.1\%) and linoleic acids (9.3-10.4\%). Multivariate regression analysis showed that oxidative stability was affected positively by hydroxytyrosol, tyrosol and oleic acid and negatively by polyunsaturated fatty acids.
\end{abstract}

(c) 2016 Elsevier Ltd. All rights reserved.

\section{Introduction}

The quality of virgin olive oil is equally affected by every step of production: agronomical (state of olive grove and olive fruits), technological (extraction system and malaxation conditions), and environmental (temperature and light during storage of olive oil). Use of healthy olive fruits at the time of extraction is a necessity in the production of virgin olive oils having considerable degree of chemical, nutritional and sensory characteristics. High quality raw material must be followed by the right operational choices in the production stage. Even healthy olive fruits are affected by adverse extraction conditions and result in poor quality olive oil. Type of extraction system and temperature-time combination in the malaxer, where the olives are crushed to form the oil part out of paste, are therefore significant parameters to be adjusted. Milling operation may seem to be a simple mechanical crushing process; however, it involves the act of several enzymes of olive, which play role in the overall quality of the final product (Clodoveo, 2012; Fregapane \& Salvador, 2013).

A strategic choice of appropriate agronomical parameters and processing conditions of olive fruits determine the overall degree of acceptability of olive oils. The parameters to be adjusted are var-

\footnotetext{
* Corresponding author.

E-mail addresses: olusolajolaym@iyte.edu.tr (O.S. Jolayemi), figentokatli@iyte, edu.tr (F. Tokatli), banuozen@iyte.edu.tr (B. Ozen).
}

ious. There are studies about some of the factors to evaluate their effects on the quality of olive oil. In the study of Monteleone, Caporale, Carlucci, and Pagliarini (1998), the olive ripening stage and storage, malaxation time and temperature effects on the total phenol and oxidative stability of olive oils were evaluated. In one study, the effect of three different extraction systems, maturity of olives and kneading temperature on the sterol composition was reported (Koutsaftakis, Kotsifaki, \& Stefanoudaki, 1999). BenDavid et al. (2010) investigated the effect of olive type, temperature, time, talc addition and different irrigation systems for a laboratory scale mill and determined oil yield, total phenol content and free fatty acidity. In other studies, researchers especially looked for the effect of oxygen in the head-space of malaxer and processing temperature with different cultivars (Catania, Vallone, Farid, \& De Pasquale, 2015; Servili, Selvaggini, Taticchi, Esposto, \& Montedoro, 2003).

Nutritionally, olive oil has chemical compounds, responsible for its comparatively higher quality values than other vegetable oils (Frankel, 2011). The presence of high percentage of monounsaturated oleic acid makes olive oil much less susceptible to oxidation and contributes to high stability and long shelf life. Olive oil polyphenols, the other factor for its unique characteristics, belong to different classes based on their molecular weights and structures: phenolic acids, phenyl ethyl alcohols (hydroxytyrosol and tyrosol), flavonoids, lignans and secoiridoids are the most characterized among others. Lately, the claims related to the support of 
oleic acid and phenolics of olive oil for healthy cholesterol levels and cardiovascular system were reported (EFSA, 2011, 2012).

In this study, it was aimed to determine the effects of olive variety, harvest time and malaxation temperature on phenolics, fatty acid profiles and oxidative stability of oils. To our knowledge, there are no scientific reports related to the combined effects of these three factors on minor components and quality characteristics of olive oils obtained in an industrial two-phase system. Unsupervised and supervised multivariate analysis were used in the evaluation of the data of full-factorial design to show the effect of quality variables in the differentiation of olive oils and explanation of oxidative stability index.

\section{Materials and methods}

\subsection{Olive oil samples}

Thirty six olive oil samples were obtained from Ayvalik (A, also called Edremit) and Memecik (M) varieties, which were extracted according to a general full factorial design in a two-phase continuous olive plant (Polat Machinery, Aydin, Turkey), located at Izmir Institute of Technology. The extractions were done at three malaxation temperatures $\left(27,37\right.$ and $\left.47^{\circ} \mathrm{C}\right)$ with the olive fruits harvested at their early, mid and late maturation stages, with two replications. The olives were harvested in the west coast of Anatolia. Olives of Ayvalik variety were obtained from Edremit Bay area in north of Izmir. Memecik olives were obtained from Aydin region in south of Izmir. The maturity levels of olive fruits were determined according to IOC method (International Olive Council, 2011). Maturity index of early, mid and late harvest olives were between 1.08-2.45, 3.23-3.57, and 4.21-6.43, respectively. Olive oil samples were kept in glass containers and head spaces were flushed with nitrogen prior to refrigerated storage $\left(4^{\circ} \mathrm{C}\right)$. In the text, abbreviations e, $\mathrm{m}$, and $\mathrm{f}$ are used for early, mid and late harvest olive oils, respectively.

\subsection{Chemicals}

Analytical grade reagents of Riedel-deHaen (Germany), Sigma-Aldrich (Germany) and Merck (Germany) were used in the analysis. Caffeic, p-coumaric, 3-hydroxyphenylacetic, 4hydroxyphenylacetic, 2,3-dihydroxylbenzoic, ferulic and gallic acids, hydroxytyrosol, tyrosol, luteolin, vanillin and pinoresinol standards were products of Extrasynthese (France) and Fluka (Germany). Fatty acid methyl ester (FAME) mixture containing C4-C24 (Supelco \#47885-U) was used as a reference standard for GC-FID analysis.

\subsection{Oxidative stability index (OSI)}

Automated oxidative stability test was performed using Rancimat system (873 Biodiesel, Metrohm, Switzerland). The reaction vessels containing $3 \mathrm{~g}$ of oil samples were covered and connected to the conductivity cells containing deionized water as volatile absorbent. The vessels were kept at $120^{\circ} \mathrm{C}$ within the heating blocks. The air flow rate was maintained at $20 \mathrm{~L} / \mathrm{h}$. The time taken for conductivity to experience a sharp increase was termed as the induction time $(\mathrm{h})$. The measurements were duplicated.

\subsection{Total phenol content (TPC)}

Total phenol content of the oil samples were determined by Folin-Ciocalteu spectrophotometric method as given in Montedoro, Servili, Baldioli, and Miniati (1992). The results were expressed in terms of gallic acid equivalent ( $\mathrm{mg} \mathrm{GA} / \mathrm{kg}$ oil).

\subsection{Chlorophyll \& carotenoid content}

A procedure given in Mínguez-Mosquera, Rejano-Navarro, Gandul-Rojas, Sanchez Gomez, and Garrido-Fernandez (1991) was used in the measurement of total chlorophyll and carotenoid contents of olive oils. The absorbances corresponding to chlorophyll $\left(A_{670}\right)$ and carotenoids $\left(A_{470}\right)$ were measured with a UV spectrophotometer (Shimadzu UV-2450 Tokyo, Japan) at $1.0 \mathrm{~cm}$ optical path (d). Chlorophyll and carotenoids were expressed in $\mathrm{mg} / \mathrm{kg}$ of oil:

$$
\begin{aligned}
& \text { Chlorophyll }(\mathrm{mg} / \mathrm{kg})=\left(\mathrm{A}_{670} \times 10^{6}\right) /(613 \times 100 \times \mathrm{d}) \\
& \text { Carotenoids }(\mathrm{mg} / \mathrm{kg})=\left(\mathrm{A}_{470} \times 10^{6}\right) /(2000 \times 100 \times \mathrm{d})
\end{aligned}
$$

\subsection{Peroxide value (PV) and free fatty acidity (FFA)}

PV and FFA analyses were done according to European Official Method of Analysis (European Union Commission, 1991). PV was expressed as meq $\mathrm{O}_{2} / \mathrm{kg}$ and FFA was expressed as \% oleic acid.

\subsection{Color}

The CIE color parameters ( $\mathrm{L}^{*}, \mathrm{a}^{*}$ and $\mathrm{b}^{*}$ ), chroma and hue ( $\mathrm{C}$ and $\mathrm{H})$ were calculated by using the standard illuminant D65 and $10^{\circ}$ observation angle from the UV-Visible spectra of the olive oil samples (Shimadzu UV-2450, Kyoto, Japan). Transmittance was taken over the range of $400-700 \mathrm{~nm}$ at $120 \mathrm{~nm} / \mathrm{min}$ scan speed in a plastic cell with $1.0 \mathrm{~cm}$ optical path length. The color parameters were calculated by the Shimadzu UVPC color analysis software (ver. 2.7). Values for each sample were obtained as the average of three replicates.

\subsection{HPLC analysis of phenolic compounds}

Phenolic profiles of olive oils were determined based on the procedure given in Alkan, Tokatli, and Ozen (2012). Amounts of individual phenolic compounds in olive oil were determined by an HPLC system (Agilent 1200, Santa Clara, CA, USA) equipped with photodiode array detector (DAD). A C18 column $(250 \mathrm{~mm}, 4 \mathrm{~mm}$, $5 \mu \mathrm{m}$, SGE 8211, Australia) was used in the analyses. Column temperature was maintained at $35^{\circ} \mathrm{C}$, injection volume was $20 \mu \mathrm{L}$ and mobile phase flow rate was adjusted to $1 \mathrm{~mL} / \mathrm{min}$. Mobile phases were water/acetic acid (99.8:0.2 v/v) and methanol. Initially, the mobile phases were $90 \%$ for water/acetic acid and $10 \%$ for methanol and the concentrations were changed according to a gradient profile during $85 \mathrm{~min}$. Gallic acid was used as the internal standard. Phenolic compounds were determined by using their commercial standards at two different wavelengths of 280 and $320 \mathrm{~nm}$. Fivepoint calibration curves for each standard were plotted and the results were expressed in terms of $\mathrm{mg} / \mathrm{kg}$ oil. Total phenolic acids (TPA) was defined as the summation of caffeic, p-coumaric, ferulic, 4-hydroxyphenylacetic, 3-hydroxyphenylacetic and 2,3 dihydroxylbenzoic acids.

\subsection{GC analysis of fatty acids}

Fatty acid methyl ester analyses were carried out according to European Official Methods of Analysis (European Union Commission, 1991). Esterified oil samples were examined with a GC-FID system (Agilent 6890, USA) including a split/splitless (1:50) injector. HP 88 capillary column $\left(100 \mathrm{~m}^{*} 0.25 \mathrm{~mm}^{*}\right.$ $0.2 \mathrm{~mm}$, Agilent, USA) was used. Helium with $2 \mathrm{~mL} / \mathrm{min}$ constant flow rate was the carrier medium. Injection volume and temperature was $1 \mathrm{~mL}$ and $250{ }^{\circ} \mathrm{C}$. Oven temperature was set to $140{ }^{\circ} \mathrm{C}$ 
initially and was maintained there for $10 \mathrm{~min}$. Then it was increased to $220^{\circ} \mathrm{C}$ with a rate of $3^{\circ} \mathrm{C} / \mathrm{min}$ and kept at this temperature for another $5 \mathrm{~min}$. The detector temperature was maintained at $280^{\circ} \mathrm{C}$. Peaks in standard mixture were compared with those of samples in the chromatogram and the results were expressed as percentage of FAME. The percentages of individual fatty acids, total saturated fatty acids (SFA), total monounsaturated fatty acids (MUFA), total polyunsaturated fatty acids (PUFA) and the ratio of oleic to linoleic acids (C18:1/C18:2) were reported.

\subsection{Statistical analysis}

The significance of factor effects on the chemical compounds was determined by Analysis of Variance (ANOVA) at 5\% probability level (Minitab 16.0, Minitab Inc., State College, USA). In the multivariate classification and regression analysis, data matrix $\mathbf{X}$ of size $(36 \times 40)$ contained 36 olive oil samples (n observations) and 40 measured variables ( $\mathrm{k}$ variables) were used in the multivariate analysis. The variables include: eleven individual phenols, total phenolic acids (TPA), summation of hydroxytyrosol and tyrosol as oleuropein derivatives (O-der), total phenol content (TPC), five quality parameters, five color parameters, eleven fatty acids, SFA, MUFA, PUFA, MUFA/PUFA and oleic to linoleic acid ratio (C18:1/ C18:2). The data were analyzed first by using PCA (Principal Component Analysis) to examine the natural data clustering. As a supervised technique, discriminant analysis by orthogonal projections to latent structures (OPLS-DA) was used in the classification of oil samples, in which case $Y$ is a user-created variable representing the classes of samples (such as 1 for early, 2 for mid and 3 for late harvest). The OPLS method provides a model which can separate the systematic variation in $\mathbf{X}$ matrix in two parts; a predictive part correlated to $\mathbf{Y}$ variable (class information in this case), an orthogonal part uncorrelated to $\mathbf{Y}$ (Galindo-Prieto, Eriksson, \& Trygg, 2015). The multivariate models were described with their number of components (PC), $\mathrm{R}^{2}$ as the total variance explained and $R_{C V}^{2}$ as the total variance explained in the leave-one-out cross validation. The principal components of OPLS models were given as $\mathrm{P}_{\mathrm{p}}+\mathrm{P}_{\mathrm{o}}$, where $\mathrm{p}$ and $\mathrm{o}$ stand for the number of predictive and orthogonal components, respectively. In the OPLS models, the insignificant variables were eliminated with the variable importance plots (VIP) as a feature of the SIMCA software (ver. 13.0, Umetrics, Umea, Sweden), then the models were rebuilt with the most significant ones.

\section{Results and discussion}

\subsection{Chemical parameters}

The quality parameters and chemical compositions of oil samples are given in Tables 1-3. The most significant factors with respect to chemical composition and quality were found as olive type, maturity level (harvest time) and their interaction $(p<0.05)$, based on ANOVA results of full factorial experimental data. The malaxation temperature and its interactions, on the other hand, were found significantly effective only for some phenolic compounds such as hydroxytyrosol, tyrosol, pinoresinol, p-coumaric acid and PV, and insignificant for fatty acid compositions. The changes in OSI, TPC and linoleic acid were not significant. The results of univariate analysis in terms of p-values are given as Supplementary data (Tables S1-S3) for all chemical characteristics.

It was observed that FFA values of Ayvalik oils slightly increased with the malaxation temperature unlike Memecik oils. High temperatures do not necessarily lead to an increase in hydrolytic activity of lipase enzyme and subsequent increase in FFA. Decrease in FFA of olive oils at temperatures above $35^{\circ} \mathrm{C}$ was also reported elsewhere (Boselli, Di Lecce, Strabbioli, Pieralisi, \& Frega, 2009; Clodoveo, Hbaieb, Kotti, Mugnozza, \& Gargouri, 2014; Panzanaro, Nutricati, Miceli, \& De Bellis, 2010). Statistically, olive oils of the two varieties were significantly different in PV with Ayvalik having lower peroxide value range (7.74-17.06 meq $\mathrm{O}_{2} / \mathrm{kg}$ ) than Memecik (13.36-25.33 meq $\mathrm{O}_{2} / \mathrm{kg}$ ). Although no correlation was detected between PV and FFA values, Memecik oils generally had higher values of both parameters. The change of PV with respect to olive type and temperature was found significant ( $\mathrm{p}$-value $<0.01$ and $\mathrm{p}$-value $<0.05$, respectively). Temperature of malaxation influenced PV of the oil as there was an increasing trend with temperature. This observation is more evident especially for the mid and late harvest Memecik oils. Carotenoid and chlorophyll pigments of the oil samples showed differences with respect to olive variety and harvest time. The findings supported the earlier reports about decreasing pigmentation with harvest time (Criado, Motilva, Goni, \& Romero, 2007). Chlorophylls and carotenoids were in the ranges of $1.50-4.55 \mathrm{mg} / \mathrm{kg}$ and $1.11-2.86 \mathrm{mg} / \mathrm{kg}$ in Memecik oils and $1.28-2.57 \mathrm{mg} / \mathrm{kg}$ and $1.01-1.61 \mathrm{mg} / \mathrm{kg}$ for Ayvalik oils, respectively. The decrease in the pigment concentrations of Ayvalik oils with ripening was not as sharp as in other samples. Similarly, color parameters of oils depend mainly on harvest time and olive variety. Memecik oils increased in lightness $\left(\mathrm{L}^{*}\right)$ with harvest time as the chromatic parameters $a^{*}$ and $b^{*}$ decreased.

The fatty acid compositions of the olive oils were significantly affected by olive type and harvest time. Oleic acid (C18:1) content of the olive oil samples was observed in the range of $70.97-75.16 \%$. An increase in oleic acid during olive fruit maturation was reported in another study (Beltran, Del Rio, Sanchez, \& Martinez, 2004). Such trend was also observed in Memecik oils, as the oleic acid content in the early harvest was found lower than the mid and late harvest oils. Linolenic acid (C18:3) content changed significantly with respect to olive type and harvest time. Ayvalik olive oils had linolenic acid in the range of $0.57-0.72 \%$, in which the late harvest oils had higher values. Conversely, Memecik olive oils had higher linolenic acid contents $(0.72-0.94 \%)$ with no particular differences among harvests. Lipoxygenase enzyme prefers linolenic acid as one of its substrates during hydroperoxide generation (Tamborrino et al., 2014), which implies that oils of high polyunsaturated fatty acids may likely have elevated PV. This can also explain higher PV of Memecik oils. Palmitic acid (C16:0) responsible for over $90 \%$ of saturated fatty acid composition of olive oil, ranged between 11.50 and $14.87 \%$. Ayvalik oils had significantly higher saturated fatty acids than Memecik olive oils, similar to the previous observations (Gurdeniz, Ozen, \& Tokatli, 2010). As reported by Manai-Djebali et al. (2012), olive oils of high SFA contents are expected to be less prone to oxidation than those high in PUFA. However, the OSI values of olive oils were not found significantly different, even though the FFA and PV of Ayvalik oils were lower than Memecik oils.

Comparison of TPC and oleuropein derivatives as hydroxytyrosol and tyrosol is shown in Fig. 1. The decrease in total phenol content with respect to harvest time is seen in Memecik oils. There is no significant difference between hydroxytyrosol contents of oils obtained from both varieties $(0.08-2.29 \mathrm{mg} / \mathrm{kg}$ Ayvalik and $0.24-$ $2.65 \mathrm{mg} / \mathrm{kg}$ Memecik) as shown in Tables 2 and 3, but difference is highly significant with respect to harvest time and malaxation temperature. High hydroxytyrosol content of oils obtained at $47^{\circ} \mathrm{C}$ is in agreement with the statement, which claimed that oleuropein degradation was enhanced during malaxation and hydrolytic enzyme $\beta$-glucosidase and esterase were released leading to the production of hydroxytyrosol and tyrosol (Taticchi et al., 2013). Protection of LDL particles from oxidative damage and maintenance of normal blood HDL-cholesterol concentration has been linked to a daily consumption of $5 \mathrm{mg}$ hydroxytyrosol and/or its derivatives per $20 \mathrm{~g}$ of olive oil by European Food Safety Authority 
Table 1

Quality parameters (mean \pm SD) of Ayvalik and Memecik olive oils at three harvest times (early, mid and late) and malaxation temperatures $\left(27{ }^{\circ} \mathrm{C}, 37^{\circ} \mathrm{C}\right.$ and $47^{\circ} \mathrm{C}$ ).

\begin{tabular}{|c|c|c|c|c|c|c|c|c|c|}
\hline \multirow[t]{2}{*}{ Responses } & \multicolumn{3}{|l|}{ Early harvest } & \multicolumn{3}{|l|}{ Mid harvest } & \multicolumn{3}{|l|}{ Late harvest } \\
\hline & $27^{\circ} \mathrm{C}$ & $37^{\circ} \mathrm{C}$ & $47^{\circ} \mathrm{C}$ & $27^{\circ} \mathrm{C}$ & $37^{\circ} \mathrm{C}$ & $47^{\circ} \mathrm{C}$ & $27^{\circ} \mathrm{C}$ & $37^{\circ} \mathrm{C}$ & $47^{\circ} \mathrm{C}$ \\
\hline \multicolumn{10}{|c|}{ Ayvalik olive oils } \\
\hline TPC & $116.7 \pm 23.0$ & $101.3 \pm 29.3$ & $84.12 \pm 0.35$ & $94.65 \pm 14.6$ & $98.96 \pm 8.3$ & $189.8 \pm 96.6$ & $191.3 \pm 111$ & $181 \pm 104$ & $83.4 \pm 3.80$ \\
\hline FFA & $1.0 \pm 0.01$ & $0.83 \pm 0.0$ & $1.60 \pm 0.08$ & $0.63 \pm 0.0$ & $0.56 \pm 0.01$ & $1.04 \pm 0.31$ & $1.96 \pm 0.23$ & $0.98 \pm 0.01$ & $1.15 \pm 0.36$ \\
\hline PV & $7.74 \pm 2.57$ & $6.50 \pm 0.13$ & $15.19 \pm 1.99$ & $12.72 \pm 3.01$ & $11.35 \pm 4.3$ & $13.36 \pm 3.9$ & $17.06 \pm 4.8$ & $9.16 \pm 0.01$ & $11.93 \pm 3.31$ \\
\hline OSI & $5.75 \pm 0.71$ & $6.96 \pm 0.21$ & $4.25 \pm 0.57$ & $4.60 \pm 0.04$ & $5.20 \pm 0.24$ & $5.89 \pm 1.08$ & $5.37 \pm 0.25$ & $5.20 \pm 0.01$ & $5.52 \pm 0.01$ \\
\hline Chl & $2.12 \pm 0.76$ & $2.57 \pm 0.31$ & $2.39 \pm 0.14$ & $1.67 \pm 0.91$ & $1.31 \pm 0.06$ & $1.51 \pm 0.11$ & $1.30 \pm 0.35$ & $1.28 \pm 0.11$ & $1.34 \pm 0.34$ \\
\hline Car & $1.43 \pm 0.35$ & $1.61 \pm 0.42$ & $1.45 \pm 0.33$ & $1.17 \pm 0.31$ & $1.01 \pm 0.21$ & $1.29 \pm 0.03$ & $1.04 \pm 0.08$ & $1.33 \pm 0.31$ & $1.05 \pm 0.12$ \\
\hline $\mathrm{L}^{*}$ & $87.5 \pm 2.57$ & $88.4 \pm 3.08$ & $91.1 \pm 2.64$ & $92.34 \pm 0.92$ & $94.97 \pm 3.53$ & $90.84 \pm 2.09$ & $87.14 \pm 0.74$ & $87.68 \pm 0.46$ & $87.77 \pm 5.77$ \\
\hline$a^{*}$ & $-1.88 \pm 1.24$ & $-2.53 \pm 1.05$ & $-3.02 \pm 0.11$ & $-3.14 \pm 1.12$ & $-4.30 \pm 0.18$ & $-3.20 \pm 0.24$ & $-0.82 \pm 0.61$ & $-3.76 \pm 0.24$ & $-3.56 \pm 0.98$ \\
\hline $\mathrm{b}^{*}$ & $71.51 \pm 12.8$ & $72.82 \pm 0.27$ & $65.36 \pm 1.77$ & $63.17 \pm 23.1$ & $49.4 \pm 0.83$ & $59.9 \pm 5.76$ & $77.05 \pm 6.02$ & $50.32 \pm 1.35$ & $54.27 \pm 2.61$ \\
\hline $\mathrm{C}$ & $71.54 \pm 12.7$ & $72.82 \pm 0.27$ & $65.36 \pm 1.77$ & $63.26 \pm 23.0$ & $49.58 \pm 0.81$ & $60.0 \pm 5.73$ & $77.14 \pm 5.96$ & $50.32 \pm 1.34$ & $54.39 \pm 2.67$ \\
\hline $\mathrm{H}$ & $91.63 \pm 1.28$ & $92.0 \pm 0.83$ & $92.66 \pm 0.16$ & $93.25 \pm 2.21$ & $94.99 \pm 0.30$ & $93.09 \pm 0.52$ & $91.75 \pm 1.12$ & $94.30 \pm 0.30$ & $93.74 \pm 0.85$ \\
\hline \multicolumn{10}{|c|}{ Memecik olive oils } \\
\hline TPC & $154.5 \pm 30.2$ & $206.1 \pm 81.8$ & $141.0 \pm 54.6$ & $117.1 \pm 18.7$ & $124 \pm 13.7$ & $194.3 \pm 10.2$ & $115.03 \pm 74.8$ & $103.9 \pm 16.3$ & $78.45 \pm 5.13$ \\
\hline FFA & $3.77 \pm 1.53$ & $1.78 \pm 0.30$ & $1.53 \pm 0.14$ & $0.87 \pm 0.13$ & $2.17 \pm 1.65$ & $1.09 \pm 0.22$ & $1.41 \pm 0.53$ & $0.79 \pm 0.06$ & $0.84 \pm 0.06$ \\
\hline PV & $18.6 \pm 1.41$ & $16.86 \pm 1.10$ & $15.18 \pm 0.95$ & $13.64 \pm 1.29$ & $15.10 \pm 0.59$ & $18.81 \pm 9.26$ & $13.36 \pm 0.05$ & $18.07 \pm 3.10$ & $25.33 \pm 11.9$ \\
\hline OSI & $4.29 \pm 1.07$ & $5.40 \pm 0.08$ & $6.22 \pm 0.30$ & $5.89 \pm 0.46$ & $5.25 \pm 2.06$ & $7.06 \pm 0.55$ & $5.35 \pm 0.59$ & $5.63 \pm 0.63$ & $5.50 \pm 1.44$ \\
\hline Chl & $4.10 \pm 0.63$ & $3.31 \pm 0.23$ & $4.55 \pm 0.73$ & $2.72 \pm 0.66$ & $2.21 \pm 0.63$ & $2.74 \pm 0.86$ & $1.67 \pm 0.06$ & $1.50 \pm 0.13$ & $1.78 \pm 0.02$ \\
\hline Car & $2.35 \pm 0.53$ & $2.38 \pm 0.52$ & $2.86 \pm 0.59$ & $2.07 \pm 0.02$ & $1.77 \pm 0.64$ & $2.14 \pm 0.43$ & $1.30 \pm 0.18$ & $1.11 \pm 0.35$ & $1.20 \pm 0.17$ \\
\hline $\mathrm{L}^{*}$ & $82.3 \pm 2.73$ & $82.6 \pm 5.34$ & $79.21 \pm 0.34$ & $81.1 \pm 5.25$ & $86.13 \pm 1.40$ & $83.26 \pm 1.40$ & $89.95 \pm 1.32$ & $92.33 \pm 3.84$ & $89.97 \pm 4.65$ \\
\hline$a_{*}$ & $0.64 \pm 2.40$ & $1.28 \pm 1.10$ & $2.05 \pm 0.36$ & $-0.51 \pm 0.40$ & $-1.24 \pm 1.56$ & $1.13 \pm 0.69$ & $-3.56 \pm 0.13$ & $-3.21 \pm 0.43$ & $-2.87 \pm 0.25$ \\
\hline$b^{*}$ & $91.36 \pm 21.1$ & $91.28 \pm 0.77$ & $99.86 \pm 1.99$ & $83.88 \pm 10.6$ & $82.13 \pm 15.1$ & $91.64 \pm 6.38$ & $53.84 \pm 3.47$ & $55.66 \pm 0.75$ & $57.99 \pm 0.60$ \\
\hline $\mathrm{C}$ & $91.38 \pm 21.2$ & $91.29 \pm 0.76$ & $99.89 \pm 2.00$ & $83.21 \pm 10.61$ & $82.89 \pm 15.1$ & $91.65 \pm 6.38$ & $53.96 \pm 3.47$ & $55.76 \pm 0.77$ & $58.06 \pm 0.59$ \\
\hline $\mathrm{H}$ & $89.77 \pm 1.47$ & $89.19 \pm 0.70$ & $88.82 \pm 0.18$ & $90.39 \pm 0.32$ & $90.98 \pm 1.25$ & $89.27 \pm 0.48$ & $93.37 \pm 0.49$ & $93.30 \pm 0.40$ & $92.84 \pm 0.28$ \\
\hline
\end{tabular}

SD: Standard deviation of two replicates, TPC: total phenol content ( $\mathrm{mg} / \mathrm{kg}$ ), FFA: Free fatty acid (\% Oleic acid), PV: Peroxide value (meq $\mathrm{O}_{2} / \mathrm{kg}$ ), OSI: Oxidative stability index (h), Chl: Chlorophylls (mg/kg), Car: Carotenoids (mg/kg), CIE color parameters: L* (lightness-darkness), a* (greenness-redness), b* (blueness-yellowness), C (Chroma), H (Hue angle).

Table 2

Phenolic and fatty acid profiles (mean \pm SD) of Ayvalik olive oils at three harvest times (early, mid and late) and malaxation temperatures $\left(27^{\circ} \mathrm{C}\right.$, $37^{\circ} \mathrm{C}$ and $47^{\circ} \mathrm{C}$ ).

\begin{tabular}{|c|c|c|c|c|c|c|c|c|c|}
\hline \multirow[t]{2}{*}{ Responses } & \multicolumn{3}{|l|}{ Early harvest } & \multicolumn{3}{|l|}{ Mid harvest } & \multicolumn{3}{|l|}{ Late harvest } \\
\hline & $27^{\circ} \mathrm{C}$ & $37^{\circ} \mathrm{C}$ & $47^{\circ} \mathrm{C}$ & $27^{\circ} \mathrm{C}$ & $37^{\circ} \mathrm{C}$ & $47^{\circ} \mathrm{C}$ & $27^{\circ} \mathrm{C}$ & $37^{\circ} \mathrm{C}$ & $47^{\circ} \mathrm{C}$ \\
\hline \multicolumn{10}{|c|}{ Phenolics ( $\mathrm{mg} / \mathrm{kg}$ ) } \\
\hline Hyt & $0.16 \pm 0.10$ & $0.13 \pm 0.02$ & $0.08 \pm 0.01$ & $0.56 \pm 0.20$ & $0.29 \pm 0.11$ & $2.29 \pm 0.49$ & $0.89 \pm 0.14$ & $0.28 \pm 0.08$ & $0.23 \pm 0.06$ \\
\hline Tyr & $8.00 \pm 0.87$ & $6.64 \pm 2.58$ & $9.26 \pm 1.53$ & $3.46 \pm 1.59$ & $2.48 \pm 0.76$ & $5.63 \pm 1.70$ & $2.22 \pm 0.42$ & $1.19 \pm 0.34$ & $1.57 \pm 0.22$ \\
\hline $4 \mathrm{Hpa}$ & $15.4 \pm 3.35$ & $16.4 \pm 3.44$ & $11.9 \pm 2.86$ & $1.48 \pm 0.28$ & $2.66 \pm 0.08$ & $2.02 \pm 0.50$ & $3.40 \pm 0.29$ & $1.44 \pm 0.13$ & $1.34 \pm 0.40$ \\
\hline 3Нра & $1.55 \pm 0.87$ & $0.86 \pm 1.03$ & $0.88 \pm 0.93$ & $0.30 \pm 0.10$ & $0.55 \pm 0.40$ & $0.65 \pm 0.62$ & $0.27 \pm 0.08$ & $0.16 \pm 0.08$ & $0.19 \pm 0.12$ \\
\hline Caf & $0.81 \pm 0.37$ & $0.76 \pm 0.13$ & $0.46 \pm 0.05$ & $0.17 \pm 0.03$ & $0.11 \pm 0.13$ & $0.11 \pm 0.10$ & $0.12 \pm 0.04$ & $0.07 \pm 0.02$ & $0.07 \pm 0.01$ \\
\hline Pin & $1.86 \pm 0.08$ & $0.86 \pm 0.17$ & $1.59 \pm 0.23$ & $7.30 \pm 6.06$ & $2.04 \pm 0.42$ & $4.45 \pm 1.43$ & $1.82 \pm 0.58$ & $0.82 \pm 0.30$ & $0.49 \pm 0.01$ \\
\hline Dba & $0.36 \pm 0.16$ & $0.24 \pm 0.01$ & $0.19 \pm 0.08$ & $0.11 \pm 0.05$ & $0.15 \pm 0.04$ & $0.18 \pm 0.00$ & $0.12 \pm 0.06$ & $0.07 \pm 0.00$ & $0.10 \pm 0.01$ \\
\hline Vnl & $0.19 \pm 0.06$ & $0.17 \pm 0.06$ & $0.19 \pm 0.21$ & $0.18 \pm 0.12$ & $0.14 \pm 0.06$ & $0.15 \pm 0.06$ & $0.08 \pm 0.05$ & $0.07 \pm 0.03$ & $0.06 \pm 0.01$ \\
\hline $\mathrm{pCu}$ & $0.77 \pm 0.05$ & $0.49 \pm 0.19$ & $0.40 \pm 0.06$ & $0.41 \pm 0.21$ & $0.18 \pm 0.02$ & $0.54 \pm 0.09$ & $0.36 \pm 0.16$ & $0.10 \pm 0.02$ & $0.14 \pm 0.01$ \\
\hline Fer & $0.21 \pm 0.08$ & $0.18 \pm 0.01$ & $0.13 \pm 0.02$ & $0.06 \pm 0.01$ & $0.05 \pm 0.01$ & $0.07 \pm 0.03$ & $0.05 \pm 0.01$ & $0.02 \pm 0.00$ & $0.03 \pm 0.01$ \\
\hline Lut & $4.75 \pm 0.93$ & $3.65 \pm 0.27$ & $4.00 \pm 0.51$ & $5.24 \pm 0.88$ & $4.08 \pm 1.07$ & $3.47 \pm 0.67$ & $2.74 \pm 0.95$ & $2.28 \pm 0.26$ & $1.39 \pm 0.04$ \\
\hline O-der & $8.16 \pm 0.97$ & $6.76 \pm 2.60$ & $9.34 \pm 1.53$ & $4.02 \pm 1.79$ & $2.77 \pm 0.87$ & $7.91 \pm 1.22$ & $3.11 \pm 0.28$ & $1.47 \pm 0.25$ & $1.80 \pm 0.28$ \\
\hline TPA & $19.13 \pm 3.13$ & $18.94 \pm 4.55$ & $13.94 \pm 1.76$ & $2.52 \pm 0.52$ & $3.69 \pm 0.16$ & $3.57 \pm 1.14$ & $4.30 \pm 0.52$ & $1.84 \pm 0.10$ & $1.85 \pm 0.57$ \\
\hline \multicolumn{10}{|l|}{ Fatty acids (\%) } \\
\hline C16:0 & $13.63 \pm 0.17$ & $13.74 \pm 0.35$ & $14.87 \pm 0.74$ & $12.68 \pm 0.42$ & $13.06 \pm 0.04$ & $12.08 \pm 0.24$ & $12.46 \pm 0.29$ & $12.10 \pm 0.03$ & $11.87 \pm 0.41$ \\
\hline C16:1 & $0.81 \pm 0.11$ & $0.82 \pm 0.06$ & $0.82 \pm 0.03$ & $0.72 \pm 0.06$ & $0.75 \pm 0.03$ & $0.58 \pm 0.10$ & $0.64 \pm 0.02$ & $0.66 \pm 0.03$ & $0.67 \pm 0.01$ \\
\hline $\mathrm{C} 17: 0$ & $0.11 \pm 0.03$ & $0.11 \pm 0.04$ & $0.12 \pm 0.02$ & $0.14 \pm 0.01$ & $0.14 \pm 0.02$ & $0.11 \pm 0.07$ & $0.15 \pm 0.02$ & $0.15 \pm 0.02$ & $0.14 \pm 0.02$ \\
\hline C17:1 & $0.19 \pm 0.04$ & $0.19 \pm 0.06$ & $0.20 \pm 0.03$ & $0.22 \pm 0.01$ & $0.21 \pm 0.02$ & $0.16 \pm 0.07$ & $0.20 \pm 0.02$ & $0.20 \pm 0.02$ & $0.20 \pm 0.02$ \\
\hline C18:0 & $2.17 \pm 0.30$ & $2.17 \pm 0.24$ & $2.31 \pm 0.11$ & $2.60 \pm 0.03$ & $2.55 \pm 0.13$ & $2.41 \pm 0.98$ & $3.08 \pm 0.03$ & $3.02 \pm 0.11$ & $2.96 \pm 0.26$ \\
\hline C18:1 & $72.75 \pm 1.46$ & $72.81 \pm 0.69$ & $71.99 \pm 0.85$ & $71.87 \pm 0.54$ & $72.34 \pm 0.25$ & $72.80 \pm 1.53$ & $73.35 \pm 2.30$ & $71.99 \pm 0.47$ & $72.29 \pm 0.80$ \\
\hline C18:2 & $9.08 \pm 0.41$ & $8.78 \pm 0.28$ & $8.36 \pm 0.29$ & $10.41 \pm 1.31$ & $9.55 \pm 0.05$ & $10.58 \pm 0.48$ & $10.41 \pm 0.23$ & $10.27 \pm 0.04$ & $10.23 \pm 0.08$ \\
\hline C18:3 & $0.61 \pm 0.03$ & $0.62 \pm 0.07$ & $0.59 \pm 0.03$ & $0.61 \pm 0.02$ & $0.59 \pm 0.02$ & $0.57 \pm 0.17$ & $0.69 \pm 0.01$ & $0.72 \pm 0.04$ & $0.72 \pm 0.02$ \\
\hline C20:0 & $0.38 \pm 0.11$ & $0.38 \pm 0.10$ & $0.44 \pm 0.04$ & $0.44 \pm 0.05$ & $0.45 \pm 0.03$ & $0.39 \pm 0.20$ & $0.52 \pm 0.01$ & $0.51 \pm 0.03$ & $0.50 \pm 0.04$ \\
\hline C20:1 & $0.22 \pm 0.12$ & $0.26 \pm 0.09$ & $0.27 \pm 0.05$ & $0.27 \pm 0.05$ & $0.29 \pm 0.03$ & $0.20 \pm 0.17$ & $0.30 \pm 0.02$ & $0.31 \pm 0.04$ & $0.32 \pm 0.03$ \\
\hline $\mathrm{C} 22: 0$ & $0.07 \pm 0.10$ & $0.16 \pm 0.03$ & $0.07 \pm 0.10$ & $0.07 \pm 0.10$ & $0.09 \pm 0.07$ & $0.16 \pm 0.02$ & $0.13 \pm 0.02$ & $0.08 \pm 0.11$ & $0.11 \pm 0.06$ \\
\hline SFA & $16.36 \pm 0.72$ & $16.56 \pm 0.70$ & $17.81 \pm 1.01$ & $15.93 \pm 0.62$ & $16.28 \pm 0.22$ & $15.15 \pm 1.47$ & $16.33 \pm 0.20$ & $15.86 \pm 0.29$ & $15.59 \pm 0.80$ \\
\hline MUFA & $73.97 \pm 1.18$ & $74.08 \pm 0.49$ & $73.27 \pm 0.73$ & $73.08 \pm 0.67$ & $73.59 \pm 0.17$ & $73.74 \pm 1.19$ & $74.49 \pm 2.24$ & $73.16 \pm 0.37$ & $73.48 \pm 0.73$ \\
\hline PUFA & $9.69 \pm 0.44$ & $9.39 \pm 0.21$ & $8.95 \pm 0.26$ & $11.02 \pm 1.32$ & $10.15 \pm 0.02$ & $11.16 \pm 0.30$ & $11.09 \pm 0.21$ & $10.99 \pm 0.08$ & $10.95 \pm 0.06$ \\
\hline C18:1/C18:2 & $8.02 \pm 0.52$ & $8.30 \pm 0.19$ & $8.61 \pm 0.20$ & $6.96 \pm 0.92$ & $7.57 \pm 0.01$ & $6.88 \pm 0.16$ & $7.05 \pm 0.07$ & $7.01 \pm 0.07$ & $7.07 \pm 0.02$ \\
\hline MUFA/PUFA & $7.64 \pm 0.47$ & $7.89 \pm 0.12$ & $8.18 \pm 0.16$ & $6.68 \pm 0.86$ & $7.25 \pm 0.00$ & $6.61 \pm 0.07$ & $6.71 \pm 0.07$ & $6.66 \pm 0.08$ & $6.71 \pm 0.03$ \\
\hline
\end{tabular}

SD: Standard deviation of two replicates, Hyt: Hydroxytyrosol, Tyr: Tyrosol, 4Hpa: 4-hydroxyphenyl acetic acid, 3Hpa: 3-hydroxyphenyl acetic acid, Caf: caffeic acid, Pin: Pinoresinol, Dba: 2,3dihydroxylbenzoic acid, Vnl; vanillin, pCu: p-Coumaric acid, Fer: Ferulic acid, Lut; Luteolin, O-der: sum of tyrosol and hydroxytyrosol, TPA: Total phenolic

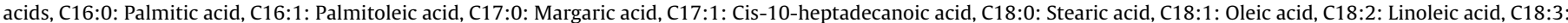

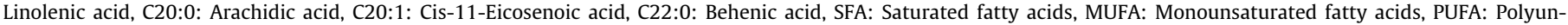
saturated fatty acids. 
Table 3

Phenolic and fatty acid profiles (mean \pm SD) of Memecik olive oils at three harvest times (early, mid and late) and malaxation temperatures $\left(27^{\circ} \mathrm{C}\right.$, $37^{\circ} \mathrm{C}$ and $47^{\circ} \mathrm{C}$ ).

\begin{tabular}{|c|c|c|c|c|c|c|c|c|c|}
\hline \multirow[t]{2}{*}{ Responses } & \multicolumn{3}{|l|}{ Early harvest } & \multicolumn{3}{|l|}{ Mid harvest } & \multicolumn{3}{|l|}{ Late harvest } \\
\hline & $27^{\circ} \mathrm{C}$ & $37^{\circ} \mathrm{C}$ & $47^{\circ} \mathrm{C}$ & $27^{\circ} \mathrm{C}$ & $37^{\circ} \mathrm{C}$ & $47^{\circ} \mathrm{C}$ & $27^{\circ} \mathrm{C}$ & $37^{\circ} \mathrm{C}$ & $47^{\circ} \mathrm{C}$ \\
\hline \multicolumn{10}{|c|}{ Phenolic profile ( $\mathrm{mg} / \mathrm{kg})$} \\
\hline Hyt & $0.28 \pm 0.01$ & $0.18 \pm 0.18$ & $0.72 \pm 0.40$ & $0.77 \pm 0.53$ & $1.16 \pm 1.01$ & $2.65 \pm 0.88$ & $0.24 \pm 0.13$ & $0.38 \pm 0.15$ & $0.36 \pm 0.12$ \\
\hline Tyr & $2.90 \pm 0.18$ & $10.17 \pm 0.62$ & $14.0 \pm 1.73$ & $12.4 \pm 5.92$ & $6.64 \pm 2.60$ & $18.0 \pm 2.01$ & $2.49 \pm 1.24$ & $3.61 \pm 1.40$ & $3.50 \pm 0.85$ \\
\hline 4Hрa & $4.88 \pm 2.78$ & $2.94 \pm 1.44$ & $6.24 \pm 0.82$ & $3.39 \pm 1.54$ & $6.32 \pm 3.70$ & $3.13 \pm 0.64$ & $2.89 \pm 2.99$ & $0.91 \pm 0.05$ & $1.01 \pm 0.26$ \\
\hline 3Нра & $0.21 \pm 0.00$ & $0.21 \pm 0.10$ & $0.20 \pm 0.04$ & $0.15 \pm 0.04$ & $0.16 \pm 0.02$ & $0.14 \pm 0.10$ & $0.22 \pm 0.03$ & $0.17 \pm 0.11$ & $0.48 \pm 0.54$ \\
\hline Caf & $0.33 \pm 0.18$ & $0.30 \pm 0.08$ & $0.40 \pm 0.06$ & $0.17 \pm 0.06$ & $0.26 \pm 0.07$ & $0.13 \pm 0.03$ & $0.13 \pm 0.11$ & $0.08 \pm 0.03$ & $0.09 \pm 0.04$ \\
\hline Pin & $12.54 \pm 1.48$ & $10.89 \pm 2.18$ & $22.3 \pm 2.45$ & $4.74 \pm 0.67$ & $8.98 \pm 2.11$ & $7.75 \pm 1.14$ & $4.29 \pm 1.24$ & $4.74 \pm 0.12$ & $4.35 \pm 0.67$ \\
\hline Dba & $0.12 \pm 0.08$ & $0.12 \pm 0.00$ & $0.31 \pm 0.06$ & $0.10 \pm 0.01$ & $0.16 \pm 0.01$ & $0.12 \pm 0.04$ & $0.08 \pm 0.03$ & $0.09 \pm 0.01$ & $0.07 \pm 0.09$ \\
\hline Vnl & $0.06 \pm 0.02$ & $0.08 \pm 0.01$ & $0.12 \pm 0.00$ & $0.20 \pm 0.18$ & $0.16 \pm 0.06$ & $0.11 \pm 0.01$ & $0.04 \pm 0.03$ & $0.05 \pm 0.01$ & $0.08 \pm 0.01$ \\
\hline $\mathrm{pCu}$ & $0.75 \pm 0.25$ & $2.05 \pm 0.75$ & $2.89 \pm 1.17$ & $0.42 \pm 0.02$ & $0.53 \pm 0.16$ & $0.87 \pm 0.23$ & $0.11 \pm 0.13$ & $0.10 \pm 0.01$ & $0.19 \pm 0.06$ \\
\hline Fer & $0.17 \pm 0.13$ & $0.19 \pm 0.10$ & $0.27 \pm 0.12$ & $0.06 \pm 0.01$ & $0.10 \pm 0.01$ & $0.08 \pm 0.03$ & $0.03 \pm 0.01$ & $0.01 \pm 0.01$ & $0.01 \pm 0.01$ \\
\hline Lut & $4.55 \pm 0.87$ & $6.64 \pm 1.47$ & $5.57 \pm 0.49$ & $0.72 \pm 0.66$ & $2.86 \pm 0.16$ & $2.88 \pm 0.26$ & $3.05 \pm 1.44$ & $3.08 \pm 0.40$ & $2.43 \pm 0.25$ \\
\hline O-der & $3.18 \pm 0.16$ & $10.35 \pm 0.43$ & $14.72 \pm 1.20$ & $13.13 \pm 5.52$ & $7.79 \pm 3.61$ & $20.65 \pm 1.12$ & $2.73 \pm 1.38$ & $3.99 \pm 1.25$ & $3.86 \pm 0.97$ \\
\hline TPA & $6.45 \pm 3.42$ & $5.81 \pm 2.47$ & $10.29 \pm 2.28$ & $4.28 \pm 1.61$ & $7.51 \pm 3.61$ & $4.47 \pm 1.07$ & $3.45 \pm 3.24$ & $1.35 \pm 0.22$ & $1.84 \pm 0.49$ \\
\hline \multicolumn{10}{|c|}{ Fatty acid profile (\%) } \\
\hline $\mathrm{C} 16: 0$ & $12.96 \pm 0.18$ & $13.47 \pm 1.10$ & $13.32 \pm 0.52$ & $12.23 \pm 0.37$ & $11.50 \pm 0.41$ & $11.60 \pm 0.08$ & $11.80 \pm 0.25$ & $11.97 \pm 0.04$ & $11.79 \pm 0.09$ \\
\hline $\mathrm{C} 16: 1$ & $0.76 \pm 0.05$ & $0.84 \pm 0.06$ & $0.88 \pm 0.00$ & $0.81 \pm 0.00$ & $0.54 \pm 0.29$ & $0.74 \pm 0.02$ & $0.85 \pm 0.05$ & $0.88 \pm 0.01$ & $0.87 \pm 0.04$ \\
\hline $\mathrm{C} 17: 0$ & $0.10 \pm 0.00$ & $0.09 \pm 0.01$ & $0.09 \pm 0.00$ & $0.07 \pm 0.00$ & $0.07 \pm 0.00$ & $0.05 \pm 0.00$ & $0.05 \pm 0.01$ & $0.04 \pm 0.00$ & $0.04 \pm 0.00$ \\
\hline $\mathrm{C} 17: 1$ & $0.15 \pm 0.02$ & $0.13 \pm 0.02$ & $0.12 \pm 0.01$ & $0.09 \pm 0.03$ & $0.11 \pm 0.05$ & $0.08 \pm 0.01$ & $0.08 \pm 0.01$ & $0.06 \pm 0.00$ & $0.06 \pm 0.00$ \\
\hline C18:0 & $2.35 \pm 0.10$ & $2.28 \pm 0.02$ & $2.50 \pm 0.07$ & $2.69 \pm 0.06$ & $2.40 \pm 0.11$ & $2.36 \pm 0.04$ & $2.45 \pm 0.10$ & $2.41 \pm 0.04$ & $2.38 \pm 0.02$ \\
\hline C18:1 & $71.63 \pm 0.36$ & $70.97 \pm 0.18$ & $71.55 \pm 0.00$ & $74.37 \pm 0.11$ & $72.73 \pm 3.53$ & $75.16 \pm 0.38$ & $72.89 \pm 0.38$ & $73.13 \pm 0.13$ & $73.88 \pm 0.31$ \\
\hline C18:2 & $10.51 \pm 0.92$ & $10.47 \pm 1.43$ & $9.72 \pm 0.57$ & $8.16 \pm 0.46$ & $11.26 \pm 4.38$ & $8.57 \pm 0.56$ & $10.17 \pm 0.09$ & $9.78 \pm 0.03$ & $9.26 \pm 0.03$ \\
\hline C18:3 & $0.76 \pm 0.17$ & $0.91 \pm 0.04$ & $0.94 \pm 0.01$ & $0.75 \pm 0.02$ & $0.78 \pm 0.06$ & $0.72 \pm 0.02$ & $0.87 \pm 0.04$ & $0.90 \pm 0.02$ & $0.89 \pm 0.06$ \\
\hline C20:0 & $0.42 \pm 0.03$ & $0.43 \pm 0.02$ & $0.48 \pm 0.01$ & $0.44 \pm 0.00$ & $0.36 \pm 0.06$ & $0.39 \pm 0.01$ & $0.44 \pm 0.02$ & $0.44 \pm 0.01$ & $0.43 \pm 0.02$ \\
\hline C20:1 & $0.29 \pm 0.02$ & $0.31 \pm 0.00$ & $0.31 \pm 0.01$ & $0.29 \pm 0.00$ & $0.26 \pm 0.03$ & $0.28 \pm 0.02$ & $0.33 \pm 0.02$ & $0.34 \pm 0.02$ & $0.33 \pm 0.03$ \\
\hline $\mathrm{C} 22: 0$ & $0.11 \pm 0.01$ & $0.12 \pm 0.00$ & $0.12 \pm 0.01$ & $0.11 \pm 0.00$ & $0.05 \pm 0.07$ & $0.09 \pm 0.01$ & $0.12 \pm 0.01$ & $0.12 \pm 0.02$ & $0.12 \pm 0.00$ \\
\hline SFA & $15.94 \pm 0.32$ & $16.39 \pm 1.15$ & $16.51 \pm 0.59$ & $15.54 \pm 0.32$ & $14.38 \pm 0.62$ & $14.49 \pm 0.13$ & $14.86 \pm 0.37$ & $14.97 \pm 0.11$ & $14.76 \pm 0.09$ \\
\hline MUFA & $72.84 \pm 0.41$ & $72.25 \pm 0.22$ & $72.86 \pm 0.00$ & $75.56 \pm 0.14$ & $73.65 \pm 3.80$ & $76.25 \pm 0.41$ & $74.15 \pm 0.32$ & $74.41 \pm 0.09$ & $75.15 \pm 0.24$ \\
\hline PUFA & $11.27 \pm 0.75$ & $11.38 \pm 1.38$ & $10.66 \pm 0.55$ & $8.90 \pm 0.44$ & $12.03 \pm 4.44$ & $9.30 \pm 0.54$ & $11.04 \pm 0.05$ & $10.68 \pm 0.01$ & $10.15 \pm 0.11$ \\
\hline C18:1/C18:2 & $6.84 \pm 0.63$ & $6.84 \pm 0.95$ & $7.37 \pm 0.43$ & $9.13 \pm 0.53$ & $7.06 \pm 3.06$ & $8.79 \pm 0.62$ & $7.17 \pm 0.03$ & $7.48 \pm 0.01$ & $7.98 \pm 0.08$ \\
\hline MUFA/PUFA & $6.48 \pm 0.47$ & $6.40 \pm 0.79$ & $6.84 \pm 0.36$ & $8.50 \pm 0.43$ & $6.63 \pm 2.76$ & $8.22 \pm 0.52$ & $6.72 \pm 0.00$ & $6.96 \pm 0.00$ & $7.41 \pm 0.11$ \\
\hline
\end{tabular}

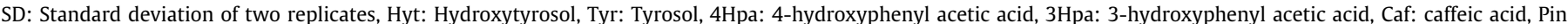

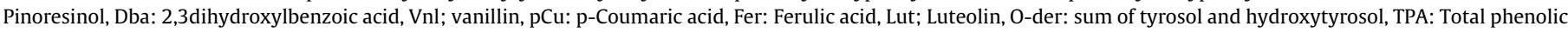

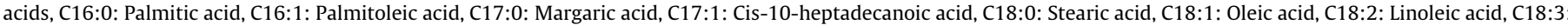

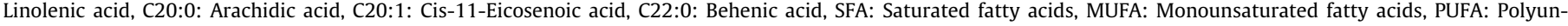
saturated fatty acids.

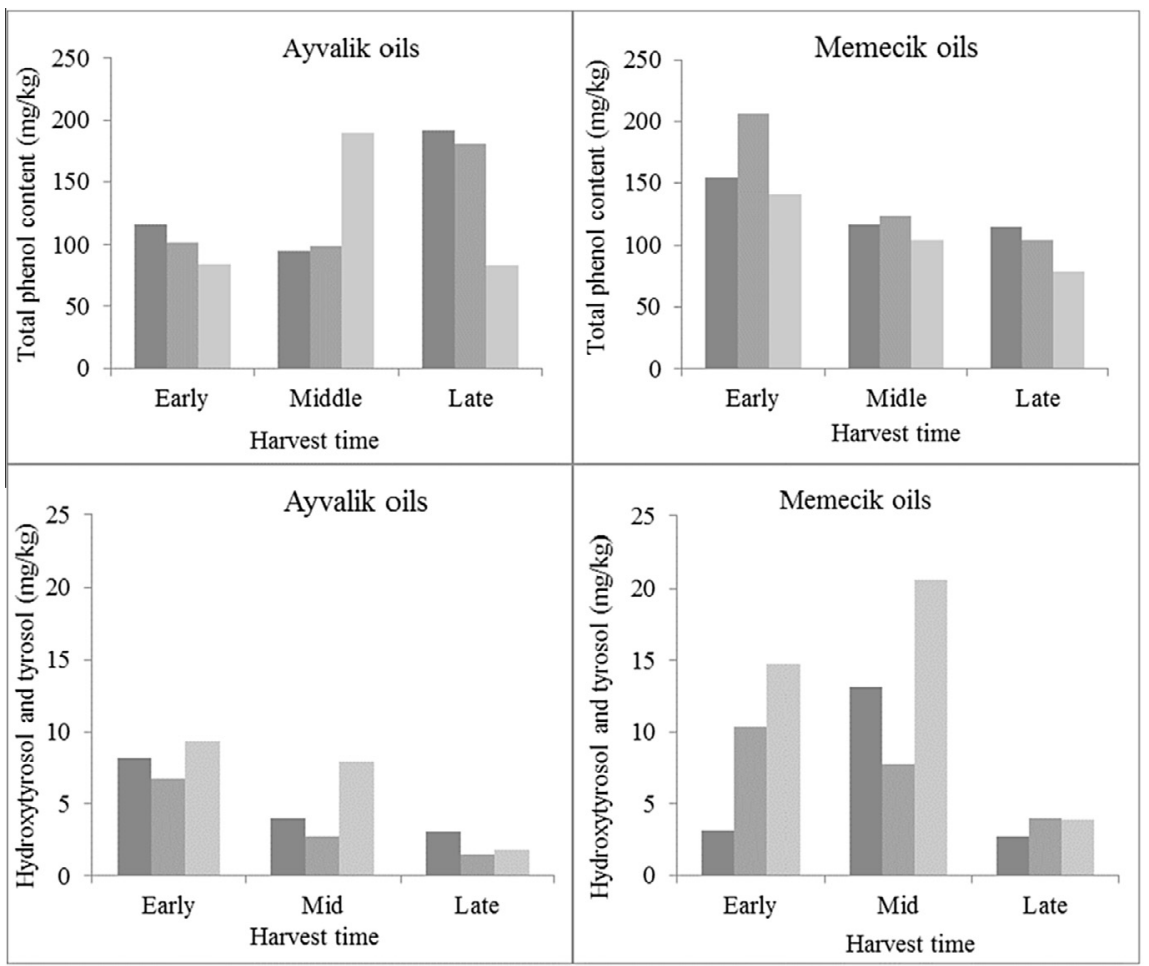

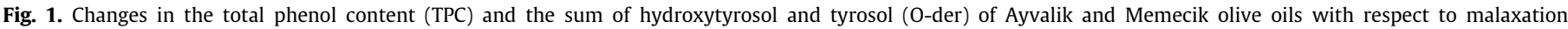
temperatures $\left(27 \square, 37 \square, 47{ }^{\circ} \mathrm{C}\right)$. 

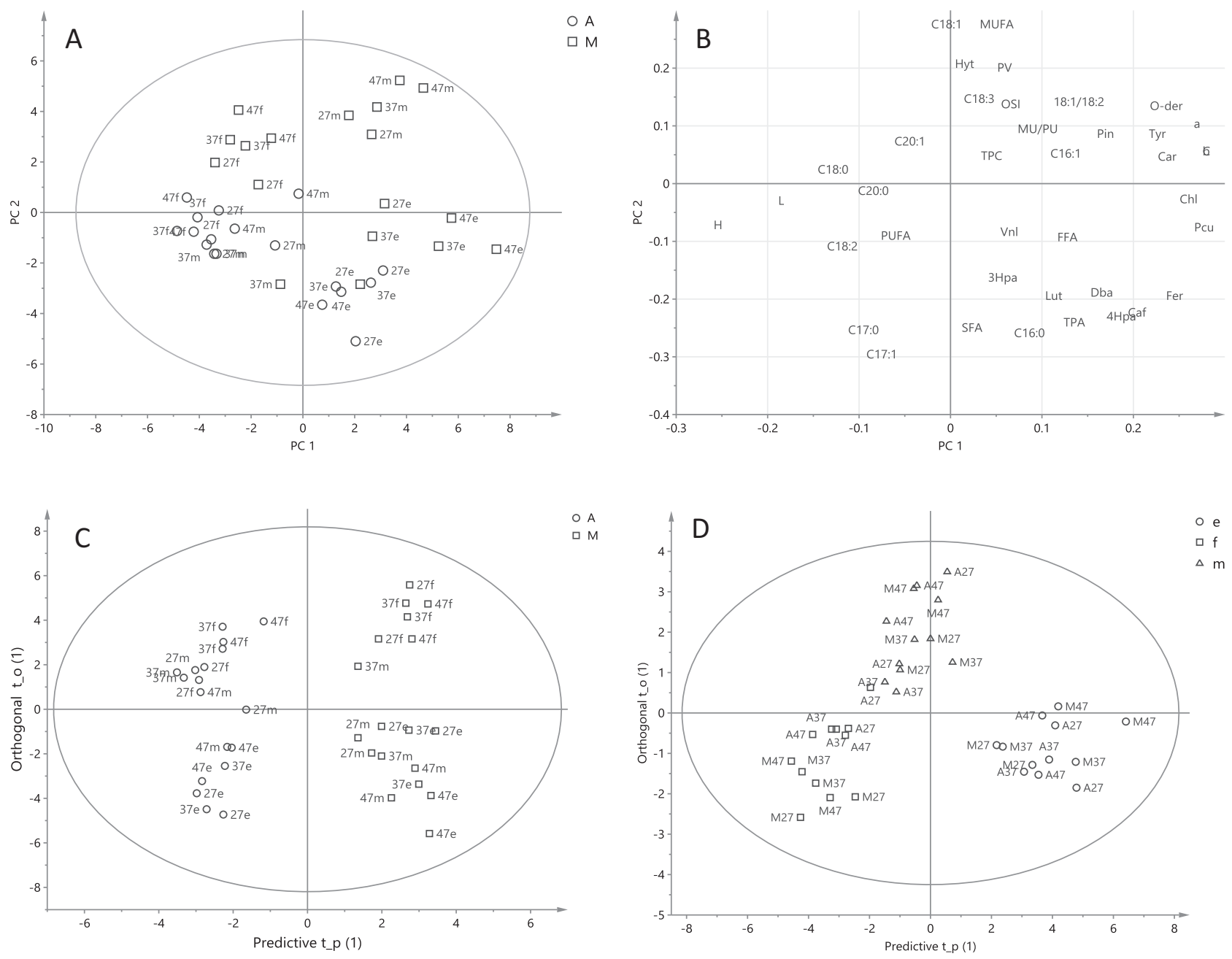

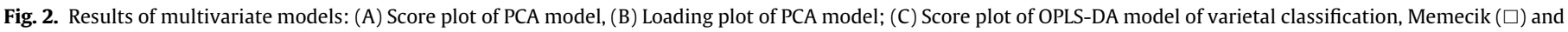
Ayvalik (O); (D) Score plot of OPLS-DA model of harvest time classification, early $(\bigcirc)$, mid $(\triangle)$ and late $(\square)$.

(EFSA, 2012). Higher amounts of oleuropein derivatives (sum of hydroxytyrosol and tyrosol) in oils were found in early and midharvest olives of both varieties (Tables 2 and 3). Tyrosol appeared to be more abundant than hydroxytyrosol in the olive oil samples. Tyrosol of Ayvalik oils linearly decreased with harvest time with the late harvest having less than $20 \%$ of the initial value. Memecik oils of early and mid-harvest had higher tyrosol contents in comparison to the oils of late harvest, with its value ranging between 2.49 and $18.0 \mathrm{mg} / \mathrm{kg}$, almost twice that of Ayvalik (1.19-9.26 mg/ $\mathrm{kg})$. This finding implies that Ayvalik and Memecik olives obtained within early to mid-harvest, and processed at $47^{\circ} \mathrm{C}$ are expected to contain higher amounts of tyrosol. Pinoresinol is an important phenolic compound belonging to the group called lignans. According to Rigane, Ayadi, Boukhris, Sayadi, and Bouaziz (2013), lignans and its derivatives are the main phenolics of olive seed. They might be present in the oil due to the breaking of pits during crushing (Cecchi et al., 2013). Pinoresinol is the most abundant phenolic compound in Memecik olive oil samples (Table 3). There was a decline in the amount of pinoresinol in Memecik oils from early harvest to late, whereas no significant change was observed in the Ayvalik oils. Pinoresinol was significantly affected by the main factors and their interactions. In the study of Taticchi et al. (2013), olive oils of different varieties were different in their pinoresinol contents, however, no significant difference was detected with respect to malaxation temperature. Studies show that olive drupes are rich in luteolin, which may exist in the form of luteolin and luteolin-7-glucosides. The presence of luteolin in the hydrophobic oil phase may be explained by the amphiphilic nature of its glucoside derivatives (Bendini et al., 2007). Luteolin was significantly affected by all factors and their interactions. The highest values of luteolin were observed at mid-harvest for Ayvalik and early harvest for Memecik oils. Luteolin content of oil of early harvest Memecik was more than twice that of mid and late harvest. Unlike another report about the relative stability of flavones and lignans with the malaxation temperature (Boselli et al., 2009), Ayvalik oils obtained at $27^{\circ} \mathrm{C}$ was significantly higher in luteolin compared to oils obtained at malaxation temperatures of 37 and $47^{\circ} \mathrm{C}$. Memecik oils obtained at $37^{\circ} \mathrm{C}$ had better luteolin content in all the harvest seasons. Total phenolic acids (TPA) of oil samples was significantly affected by variety, harvest time and combined effect of both. Ayvalik oils had higher TPA (1.86-19.13 mg/kg) than Memecik oils (1.43-10.38 mg/kg).

\subsection{Multivariate analysis}

Statistical models built with the data of quality parameters, phenolic compounds and fatty acids were used to highlight the differences/similarities among oil samples with respect to the significant factors. According to a PCA model with 5 PCs, $\mathrm{R}^{2}$ of 0.81 and $R_{C V}^{2}$ of 0.53 , the distribution patterns of oils is clearly with respect to 

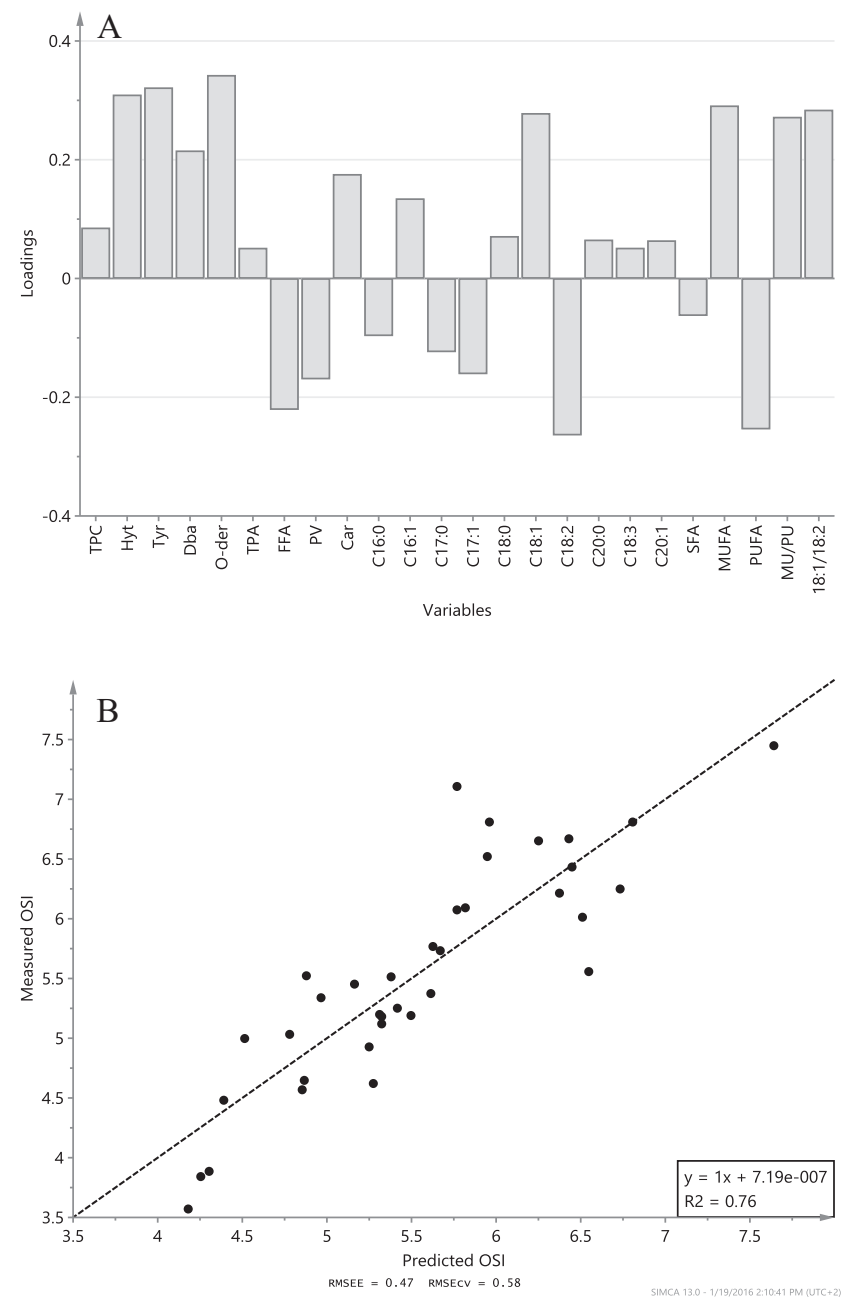

Fig. 3. Prediction results of OSI with OPLS model. The loading weights of chemical variables of the regression model (A) and scatter plot of predicted to measured values (B). RMSEE: root mean square error of estimation; $\mathrm{RMSE}_{\mathrm{CV}}$ : root mean square of the cross validation.

harvest time and olive variety rather than malaxation temperature (Fig. 2A). The most recognizable pattern in this unsupervised model is that the early harvest Ayvalik oils are different than mid and late harvest oils. Early harvest Ayvalik oils are mainly defined by their comparatively higher amount of phenolic acids and saturated fatty acids according to the loading plot (Fig. 2B). In case of Memecik oils, the early harvest oils show similar characteristics as Ayvalik oils of the same harvest, however mid harvest Memecik oils have differences from other olive oils as they are clustered in the upper right quarter in the score plot (Fig. 2A). The presence of vanillin and phenolic acids such as $4 \mathrm{Hpa}, 3 \mathrm{Hpa}$, caffeic, ferulic, and Dba can contribute to stability and nutritional quality of Ayvalik oils. Even though phenolic acids can be relatively less potent to oxidative stability compared to oleuropein derivatives (hydroxytyrosol, tyrosol etc.) and secoiridoids compounds, they can form a protective action against to oxidation (Servili et al., 2013). The lower linolenic acid content of early and mid-harvest Ayvalik oils can be considered as another factor for the stability. The oxidative stability parameters and health implicative variables such as phenolic compounds, pigments, oxidative stability index, and also degree of saturation are all localized at the right part of the loading plot corresponding to the characteristics of early or mid-harvest of both olive varieties. The late harvest oil samples located in the upper part of the control ellipse can be characterized with certain fatty acids such as linoleic, stearic and arachidic acids and higher PV.

An OPLS-DA model with $1+2$ PCs, $R^{2}$ of 0.945 and $R_{C V}^{2}$ of 0.855 was built to show the classification of oils with respect to cultivar. As can be seen in the score plot of the model, Ayvalik and Memecik oil samples are clustered in different parts of the control ellipse (Fig. 2C). In case of Memecik oils, the early and mid-harvest oils separated themselves in the lower right quarter of the ellipse with their typical properties such as higher contents of tyrosol, pinoresinol, pcoumaric acid, color pigments and different color characteristics. The oxidative stability of Memecik oils, despite their significant high peroxide values, free fatty acids, and linolenic acids content, can be explained by the defensive effect of phenols against lipid oxidation. The classification of olive oils with respect to harvest time is shown in the score plot of OPLS-DA model with $2+2 \mathrm{PCs}, \mathrm{R}^{2}$ of 0.82 and $\mathrm{R}_{\mathrm{CV}}^{2}$ of 0.62 (Fig. 2D). Early harvest olive oils were distantly located from other oils. And yet, the mid and late harvest olive oils formed clusters of their own. There is no differentiation of malaxation temperatures in the clusters of two models (Fig. 2C and D). In terms of high phenolic content, pigment concentration and stability (high OSI, low PV and FFA), early harvest oils of both varieties and Memecik oils from mid-harvest olives showed similar characteristics irrespective of malaxation temperature between 27 and $47^{\circ} \mathrm{C}$. The comments on the high malaxation temperature of $47^{\circ} \mathrm{C}$ are limited to this statement, since sensory analysis was not performed on the olive oils.

In order to explain the effects of chemical and quality parameters on oxidative stability, a regression model for OSI was built with OPLS technique. According to VIP values of the model, chlorophyll content, pinoresinol, vanillin, $3 \mathrm{Hpa}, 4 \mathrm{Hpa}$ and C18:0 were not effective in modelling of OSI and removed from the data set (VIP less than 0.3 ). The OPLS model was rebuilt with the remaining variables. The loadings of predictive component of the model with 1 +2 PCs, $R^{2}$ of 0.76 and $R_{\mathrm{CV}}^{2}$ of 0.6 are given in Fig. 3 (loadings bar plot and regression plot for OSI). With respect to their weights in the regression model, total concentration of hydroxytyrosol and tyrosol (O-der), the ratio of oleic/linoleic acids and oleic acid have positive effects on OSI of olive oils. The high concentrations of free fatty acid, peroxide value, linoleic acid, and total polyunsaturated fatty acids have adverse effects on the stability. It was also observed that high total phenol content did not necessarily cause stability, but rather carotenoids and individual phenolic substances such as hydroxytyrosol and tyrosol did. The significant effects of hydroxytyrosol and total hydroxytyrosol and tyrosol concentrations on the oxidative stability were reported by other researchers (Allalout et al., 2009; Uncu \& Ozen, 2015).

\section{Conclusion}

The degree of maturation as early, mid and late harvest olives was the most significant factor affecting chemical and quality profiles of olive oils from Ayvalik and Memecik varieties. The malaxation temperatures between 27 and $47^{\circ} \mathrm{C}$ were found significant on the changes of hydroxytyrosol, tyrosol, pinoresinol, p-coumaric acid contents and peroxide values. It was also observed that the interaction between olive variety and harvest time was significant, that is the oils of different types had different characteristics with respect to harvest time. As a result of multivariate classification models, it was concluded that early and mid-harvest Memecik oils showed similar properties, and early harvest Ayvalik oils had significantly different chemical qualities than its mid and late harvest oils. The olive oils with high oxidative stability and nutritional quality could be produced from early harvest olives even at high temperatures up to $47^{\circ} \mathrm{C}$. 
Oxidative stabilities of oil samples were investigated with a multivariate regression model (OPLS) to determine the effect of chemical compositions. The sum of hydroxytyrosol and tyrosol concentrations, carotenoids and oleic acid/linoleic acid ratio had positive effects on the stability; high free fatty acid, peroxide value and linoleic acid contents might have caused a decrease on the stability parameter. Total phenol and chlorophyll contents were not found effective on the oxidative stability index.

\section{Conflict of interest}

The authors declare that they have no conflict of interest.

\section{Acknowledgments}

The authors thank Environmental Research Center and Biotechnology and Bioengineering Research Center of Izmir Institute of Technology for GC and HPLC analysis, Taris Olive and Olive Oil Cooperatives Union for providing Ayvalik and Memecik olives, and Turkey Scholarships Program for International Students (YTB) and The Federal University of Technology Akure for the Ph.D. grant for Mr. Jolayemi.

\section{Appendix A. Supplementary data}

Supplementary data (the results of ANOVA as p-values of factors) associated with this article can be found, in the online version, at http://dx.doi.org/10.1016/j.foodchem.2016.05.134.

\section{References}

Alkan, D., Tokatli, F., \& Ozen, B. (2012). Phenolic characterization and geographical classification of commercial extra virgin olive oils produced in Turkey. Journal of the American Oil Chemists' Society, 89, 261-268.

Allalout, A., Krichène, D., Methenni, K., Taamalli, A., Oueslati, I., Daoud, D., \& Zarrouk, M. (2009). Characterization of virgin olive oil from super intensive Spanish and Greek varieties grown in northern Tunisia. Scientia Horticulturae, 120, 77-83.

Beltran, G., Del Rio, C., Sanchez, S., \& Martinez, L. (2004). Influence of harvest date and crop yield on the fatty acid composition of virgin olive oils from Cv. Picual. Journal of Agricultural and Food Chemistry, 52, 3434-3440.

Ben-David, E., Kerem, Z., Zipori, I., Weissbein, S., Basheer, L., Bustan, A., \& Dag, A. (2010). Optimization of abencor system to extract olive oil from irrigated orchards. European Journal of Lipid Science and Technology, 112, 1158-1165.

Bendini, A., Cerretani, L., Carrasco-Pancorbo, A., Gómez-Caravaca, A. M., SeguraCarretero, A., Fernández-Gutiérrez, A., \& Lercker, G. (2007). Phenolic molecules in virgin olive oils: a Survey of their sensory properties, health effects, antioxidant activity and analytical methods. An overview of the last decade. Molecules, 12(8), 1679-1719.

Boselli, E., Di Lecce, G., Strabbioli, R., Pieralisi, G., \& Frega, N. G. (2009). Are virgin olive oils obtained below $27^{\circ} \mathrm{C}$ better than those produced at higher temperatures? LWT - Food Science and Technology, 42(3), 748-757.

Catania, P., Vallone, M., Farid, A., \& De Pasquale, C. (2015). Effect of $\mathrm{O}_{2}$ control and monitoring on nutraceutical properties of extra virgin olive oils. Journal of Food Engineering, 169, 179-188.

Cecchi, L., Migliorini, M., Cherubini, C., Giusti, M., Zanoni, B., Innocenti, M., \& Mulinacci, N. (2013). Phenolic profiles, oil amount and sugar content during olive ripening of three typical Tuscan cultivars to detect the best harvesting time for oil production. Food Research International, 54(2), 1876-1884.

Clodoveo, M. L. (2012). Malaxation: Influence on virgin olive oil quality. Past, present and future - An overview. Trends in Foods Science E Technology, 25(1), $13-23$.

Clodoveo, M. L., Hbaieb, R. H., Kotti, F., Mugnozza, G. S., \& Gargouri, M. (2014). Mechanical strategies to increase nutritional and sensory quality of virgin olive oil by modulating the endogenous enzyme activities. Comprehensive Reviews in Food Science and Food Safety, 13(2), 135-154.
Criado, M. N., Motilva, M. J., Goni, M., \& Romero, M. P. (2007). Comparative study of the effect of the maturation process of the olive fruit on the chlorophyll and carotenoid fractions of drupes and virgin oils from Arbequina and Farga cultivars. Food Chemistry, 100, 748-755.

EFSA (2011). Panel on dietetic products, nutrition and allergies (NDA); scientific opinion on the substantiation of health claims related to oleic acid intended to replace saturated fatty acids (SFAs) in foods or diets and maintenance of normal blood LDL-cholesterol concentrations (ID 673, 728, 729, 1302, 4334) and maintenance of normal (fasting) blood concentrations of triglycerides (ID 673 4334) pursuant to article 13(1) of regulation (EC) No. 1924/2006. EFSA Journal, 9 (4), 2043.

EFSA (2012). Panel on dietetic products, nutrition and allergies (NDA); scientific opinion on the substantiation of a health claim related to polyphenols in olive and maintenance of normal blood HDL-cholesterol concentrations (ID 1639, further assessment) pursuant to article 13(1) of regulation (EC) No. 1924/2006. EFSA Journal, 10(8), 2848.

European Union Commission (1991). Regulation EEC 2568/91 on the characteristics of olive oil and olive-residue oil and on the relevant methods of analysis. Official Journal of European Communities, 1248.

Frankel, E. N. (2011). Nutritional and biological properties of extra virgin olive oil. Journal of Agricultural and Food Chemistry, 59(3), 785-792.

Fregapane, G., \& Salvador, M. D. (2013). Production of superior quality extra virgin olive oil modulating the content and profile of its minor components. Food Research International, 54(2), 1907-1914.

Galindo-Prieto, B., Eriksson, L., \& Trygg, J. (2015). Variable influence on projection (VIP) for OPLS models and its applicability in multivariate time series analysis. Chemometrics and Intelligent Laboratory Systems, 146, 297-304.

Gurdeniz, G., Ozen, B., \& Tokatli, F. (2010). Comparison of fatty acid profiles and mid-infrared spectral data for classification of olive oils. European Journal of Lipid Science and Technology, 112, 218-226.

International Olive Council (2011). Guide for the determination of the characteristics of oil-olives, $\mathrm{COI} / \mathrm{OH} / \mathrm{Doc}$. No. 1.

Koutsaftakis, A., Kotsifaki, F. \& Stefanoudaki, E. (1999). Effect of extraction system, stage of ripeness and kneading temperature on the sterol composition of virgin olive oils. Journal of American Oil Chemists' Society, 76(12), 1477-1481.

Manai-Djebali, H., Krichene, D., Ouni, Y., Gallardo, L., Sanchez, J., Osorio, E., Daoud, D., ... Zarrouk, M. (2012). Chemical profiles of five minor olive oil varieties grown in central Tunisia. Journal of Food Composition and Analysis, 27, 109-119.

Mínguez-Mosquera, M. I., Rejano-Navarro, L., Gandul-Rojas, B., Sanchez Gomez, A. H., \& Garrido-Fernandez, J. (1991). Color-pigment correlation in virgin olive oil. Journal of the American Oil Chemists' Society, 68, 332-336.

Montedoro, G., Servili, M., Baldioli, M., \& Miniati, E. (1992). Simple and hydrolysable phenolic compounds in virgin olive oil. 1 . Their extraction, separation, and quantitative and semiquantitative evaluation by HPLC. Journal of Agricultural and Food Chemistry, 40, 1571-1576.

Monteleone, E., Caporale, G., Carlucci, A., \& Pagliarini, E. (1998). Optimization of extra virgin olive oil quality. Journal of the Science of Food and Agriculture, 77, 31-37.

Panzanaro, S., Nutricati, E., Miceli, A., \& De Bellis, L. (2010). Biochemical characterization of lipase from olive frit (Olea europaea L.). Plant Physiology and Biochemistry, 48(9), 741-745.

Rigane, G., Ayadi, M., Boukhris, M., Sayadi, S., \& Bouaziz, M. (2013). Characterisation and phenolic profiles of two rare olive oils from southern Tunisia: Dhokar and Gemri-Dhokar cultivars. Journal of the Science of Food and Agriculture, 93(3), 527-534.

Servili, M., Selvaggini, R., Taticchi, A., Esposto, S., \& Montedoro, G. (2003). Volatile compounds and phenolic composition of virgin olive oil: Optimization of temperature and time of exposure of olive pastes to air contact during the mechanical extraction process. Journal of Agricultural and Food Chemistry, 51 (27), 7980-7988.

Servili, M., Sordini, B., Esposto, S., Urbani, S., Veneziani, G., Di Maio, I., \& Taticchi, A. (2013). Biological activities of phenolic compounds of extra virgin olive olive. Antioxidants, 3(1), 1-23.

Tamborrino, A., Pati, S., Romaniello, R., Quinto, M., Zagaria, R., \& Leone, A. (2014). Design and implementation of an automatically controlled malaxer pilot plant equipped with an in-line oxygen injection system into the olive paste. Journal of Food Engineering, 141, 1-12.

Taticchi, A., Esposto, S., Veneziani, G., Urbani, S., Selvaggini, R., \& Servili, M. (2013). The influence of the malaxation temperature on the activity of polyphenoloxidase and peroxidase and on the phenolic composition of virgin olive oil. Food Chemistry, 136(2), 975-983.

Uncu, O., \& Ozen, B. (2015). Prediction of various chemical parameters of olive oils with Fourier transform infrared spectroscopy. LWT - Food Science and Technology, 63, 978-984. 